\title{
Selection for the bacterial capsule in the absence of biotic and abiotic aggressions depends on growth conditions
}

\author{
Amandine Buffet ${ }^{1}$, Eduardo P.C. Rocha ${ }^{1} \&$ Olaya Rendueles $*^{1}$ \\ ${ }^{1}$ Microbial Evolutionary Genomics, Institut Pasteur, CNRS, UMR3525, Paris, 75015, France. \\ * olaya.rendueles-garcia@pasteur.fr
}

Keywords: capsule serotype/evolution/biotic stress/diversifying selection/

\section{SUMMARY}

Extracellular capsules protect the cell against both abiotic and biotic stresses such as bacteriophages and the host immune system. Yet, it is unclear if capsules contribute to fitness in the absence of external aggressions, in spite of the cost of production. Here, we enquire if there are conditions favouring the presence of the capsule in Klebsiella, where it is a major virulence factor. We shortly experimentally evolved 19 different strains, and show that small changes in growth media have a strong impact on the maintenance of the capsule. Competitions with capsule mutants in nine different strains showed that capsules provide $\sim 20 \%$ of fitness advantage in nutrient-poor conditions, due to faster growth rates and higher yields. In contrast, the capsule was readily lost in nutrient-rich media. The growth environment, as well as the capsule serotype, strongly influenced the role of the capsule in major virulence traits like hypermucoviscosity and biofilm formation. Our data shows that the capsule is selected for in situations lacking abiotic, but also biotic stresses and further supports that the capsule role in virulence may be a by-product of adaptation outside the host, hinting that it may have important roles in bacterial physiology yet to be discovered.

\section{SIGNIFICANCE}

Bacterial capsules are a wide-spread virulence factor that limits efficacy of antimicrobial therapy. Whereas most studies focus on the role of the capsule in pathogenesis, very few have addressed the conditions under which the capsule is primarily selected for. Here, we show that small changes in growth media have a strong impact in the maintenance of the capsule and the 
fitness advantage they confer. Our results raise the question whether conditions lacking biotic or abiotic stresses, in addition of selecting for the maintenance of the capsule, can also play a role in selecting for serotype variation. Our results further support that the role of the capsule in virulence may be a by-product of adaptation outside the host, hinting that there may be other functionalities yet to be discovered for it. 


\section{INTRODUCTION}

Most environments in which bacteria thrive are complex and can be temporally or spatially heterogeneous, both in their abiotic ( $\mathrm{pH}$, nutrients, chemicals...) or biotic compositions (niche invasions, extinction events). This can represent an evolutionary challenge for microbes (Levins 1968, Meyers and Bull 2002). In order to survive, bacterial species can either move to less stressful territories, adapt to the new circumstances or resist until conditions are suitable (Rittershaus et al 2013). Notably, bacteria can produce a thick extracellular layer - the capsule - that has been reported to enhance survival and increase tolerance to external aggressions (Ophir and Gutnick 1994, Tipton et al 2018). Extracellular capsules constitute the first barrier between the cell and its environment and are encoded in half of the bacterial genomes, in some archaea and yeast (Rendueles et al 2017). Capsules fulfill many roles in bacterial physiology. They have been associated with resistance to abiotic stresses such as UV light and desiccation (Ophir and Gutnick 1994, Tipton et al 2018). However, they are mostly studied for their role enhancing survival under biotic stresses, including resistance to grazing protozoa (Jung et al 2007), antibiotics (Fernebro et al 2004, Geisinger et al 2019), antimicrobial peptides (Band and Weiss 2015, Campos et al 2004), and host immune defences, such as human serum and phagocytes (Podschun and Ullmann 1992, Tomas et al 1986, Williams et al 1983).

The majority of capsules belong to the so-called Group I or Wzx/Wzy-dependent capsules (Rendueles et al 2017). These capsules are high molecular weight polysaccharides made up of repeat units of oligosaccharides. The different possible oligosaccharide combinations and residue modifications lead to different serotypes. Serotype diversity, even within-species, is very large. This genetic diversity has been particularly studied in facultative pathogens such as Streptococcus pneumoniae (Bentley et al 2006), Campylobacter jejuni (Guerry et al 2012), and Acinetobacter baumanii (Giguere 2015). In Klebsiella pneumoniae, there are at least 77 serologically-defined capsule serotypes (Mori et al 1989, Orskov 1955, Podschun and Ullmann 1998), and more than 130 serotypes identified through comparative genomics

30 (Follador et al 2016, Wyres et al 2016). This serotype diversity results from the faster evolutionary rates of capsular loci compared to those of the rest of the genome. The former has been shown to occur by homologous recombination (Mostowy et al 2017, Wyres et al 2015) and horizontal gene transfer (Croucher et al 2015, McBride et al 2007, Thrane et al 
2015). As a result, the same capsular serotype can be found in multiple lineages of $K$.

pneumoniae and a monophyletic clade can include distinct serotypes (Wyres et al 2015, Wyres et al 2019). Selection for rapid variation of capsule serotypes is thought to result from biotic stresses, notably because phages and the human adaptive immune system target the capsule in a serotype specific manner, which favours the emergence of novel serotypes (Mostowy and Holt 2018). This process can be accelerated by exposure to vaccines which target specific capsule serotypes (Croucher et al 2013, Guerry et al 2012, Lamb et al 2014).

Many studies have assessed the role of the capsule during pathogenesis, yet little is known about the primary selective forces that underlie the emergence and maintenance of the capsule when bacteria are not under biotic stress. Since capsules are costly to produce, if they are selected mainly to protect bacterial cells from phages, immune systems, or specific stresses, they should be readily lost in the absence of these challenges. Consequently, serial passaging of Klebsiella in lithium chloride peptone water, as well as aging of cultures, readily leads to the emergence of non-capsulated clones (Randall 1939), indicating that the capsule can be easily lost.

To further investigate the conditions where the capsule is advantageous for the cell, we study bacteria from the genus Klebsiella. This genus is composed of ubiquitous free-living and host-associated bacteria able to colonize a large range of environments (Bagley 1985, Wyres et al 2020), due to a diverse metabolism (Blin et al 2017) and the presence of a Wzx/Wzydependent capsule (Follador et al 2016). Klebsiella spp., and more particularly $K$. pneumoniae, are opportunistic pathogens and major multi-drug resistant (MDR) bacteria that cause both community-acquired and hospital-acquired infections in humans (Bengoechea and Pessoa 2019, Caneiras et al 2019, Lee et al 2017). Most isolated Klebsiella have a capsule, and the latter is regarded as the major virulence factor of the genus (Paczosa and Mecsas 2016). To test the maintenance of capsule production in several growth conditions, including host-related environments, we selected nineteen Klebsiella strains representative of the genetic diversity of the K. pneumoniae species complex (Blin et al 2017). These strains have different capsule serotypes (K-locus) and O-antigens (O-locus), and include a closely-related K. variicola (strain \#24), and two K. quasipneumoniae subsp. similpneumoniae (strains \#44 \& \#214) (Table S1). We generated a panel of nine capsule mutants to address the precise fitness effects of the capsule in a range of conditions. Finally, we explored the importance of the serotypes and the genetic background of the strain in driving capsule-related phenotypes. The 
integration of these results revealed that the capsule can be selected in the absence of biotic stresses.

\section{RESULTS}

\section{The capsule is lost in nutrient rich environments}

Early reports (Julianelle 1928, Randall 1939) and our laboratory observations reveal that

serial passaging of Klebsiella cultures in rich laboratory media (LB) generates spontaneous mutants lacking the capsule (de Sousa et al 2020). This strongly suggests that capsule has a negative impact on fitness in laboratory environments. To understand the fitness cost of capsule in the absence of biotic pressures, we serially passaged 19 different Klebsiella strains belonging to six different serotypes (at least three strains per serotype), among which the virulence-associated KL1, KL2 and the emergent KL107 associated to multi-drug resistance. We then measured the natural emergence of non-capsulated mutants after $c a$. 20 generations in five different growth media (see Methods). Artificial sputum medium (ASM) (Fung et al 2010) and artificial urine medium (AUM) (Brooks and Keevil 1997) mimic host-related nutritional conditions. The remaining are typical laboratory media with decreasing levels of nutrients: rich LB medium, minimal medium supplemented with $0.2 \%$ of glucose (M02), and minimal medium supplemented with $0.05 \%$ of glucose (M005). Beforehand, we verified that the strains were able to produce a capsule in the different growth conditions by direct microscopic observation and by capsule quantification using the uronic acid method (Methods, Figure S1). To initiate the experiment, we grew three independent clones of each strain for 16 hours in LB and then diluted (1:100) into each growth media. All 19 strains were then serially transferred into fresh media every 24 hours for three days. Although each growth media has different carrying capacities, cultures were always diluted to 1:100 and after $24 \mathrm{~h}$ of growth and each media was saturated with cells in late stationary phase. This ensured that in all growth media, the different populations underwent a similar number of generations $(\sim 20)$.

Non-capsulated mutants emerged after three days, in 111 of the 290 independently evolved populations (38\%) (Figure 1). Most ASM (74\%) and LB (89\%) lineages showed capsule mutants, and these accounted for 85 of the 111 populations with non-capsulated mutants. In contrast, non-capsulated mutants were very rarely observed in the other three growth media 
in the growth media that drove the maintenance of the capsule, rather than the opposition between host-related environments versus lab environments. Indeed, it was in the nutrient rich media (ASM and LB), where the capsule was readily lost, whereas it was consistently maintained in the three nutrient poor media (M02, M005 and AUM). To further confirm this, we allowed the experiment to run for 30 days in LB and M02 for a KL2 strain (\#26). The three replicate populations that evolved in M02 remained capsulated whereas all populations evolving in LB rapidly and irreversibly lost their capsule (Figure S2).

Some strains seem more prone to lose the capsule. An extreme example concerns strain \#62

110 for which we found capsule mutants in all fifteen independently-evolved populations across the five different growth media. We tested if these differences could be due to the amount of capsule produced by each strain, but the frequency of non-capsulated mutants does not correlate with the amount of capsule produced by each strain before the evolution experiments $(\mathrm{P}>0.05$, Spearman's rank correlation). We tested the relevance of both

115 serotype and growth media on the emergence of capsule mutants using a generalized linear model $\left(\mathrm{R}^{2}=0.39\right)$. This revealed that the emergence of capsule mutants was both dependent on the growth media $(\mathrm{P}<0.001)$ and on the serotype $(\mathrm{P}<0.02)$. This suggests that the capsule is deleterious under rich media and selected for in poor media, a factor that depends at a lesser extent on the capsule serotype.

120

\section{The capsule provides a fitness advantage in nutrient poor environments.}

To precisely assess the costs and benefits of the capsule across growth media, we sought to perform competition experiments between capsulated (Cap+) and their respective isogenic non-capsulated (Cap-) mutants. We thus undertook the construction of in-frame and

125 markerless deletions of $w z a$, the outer membrane exporter of the capsule, a well-annotated and essential core gene of the capsule operon. We used the allelic recombination strategy and aimed at deleting wza in 15 different strains from different serotypes, for which we had easily obtained $\operatorname{rcs} B$ in-frame deletion mutants. ( $\operatorname{RcsB}$ is a capsule regulator and mutants have a reduced capsule production (Dorman et al 2018, Wacharotayankun et al 1992)). We obtained

$130 w z a$ deletion mutants for five strains, from several serotypes (see Table S1). From these double-recombination events, we isolated both a mutant and a wild type. This ensures that the phenotype was directly due to the $w z a$ deletion and not to other off-target mutations that could have accumulated in the genome during the mutant generation, and thus present in both the 
135 the $\Delta w z a$ mutant and the associated wild type from the same double-recombination event. After several attempts, we obtained wza deletion mutants in four additional strains, but Illumina sequencing revealed off-target compensatory mutations only present in the mutant (and not in the associated wild type) (see Methods). In the main text, we present statistics and figures for all strains, and in supplemental material, for the five clean mutants. Microscopic

140 observation and uronic acid quantification confirmed that all nine $\Delta w z a$ mutants lack a capsule (Figure S3). For the remaining seven strains, no $\Delta w z a$ mutants were obtained despite several attempts.

To assess the competitive indexes, or fitness, of the mutant strains, we performed direct 145 competition experiments between wild type strains and their respective $\Delta w z a$ mutant strains. The strains were initially mixed in a 1:1 ratio and allowed to compete in all five growth media for a 24-hour period. We estimated that the natural emergence of capsule mutants in rich media is rare during the first 24 hours and is thus negligible in the interpretation of our results. Competition experiments showed a significant effect of the environment in the fitness

150 of the Cap + strains (Figure 2, Kruskal-Wallis, $\mathrm{P}=0.0046, \mathrm{~N}=8$ ). In this analysis, we excluded strain \#55 due to a growth deficiency of the $\Delta w z a$ mutant. Overall, we observe a marginal decrease of Cap+ genotypes in nutrient rich media $(\mathrm{P}=0.07$ and $\mathrm{P}=0.05$ in $\mathrm{LB}$ and ASM, respectively, one-sided t-test, difference of 1). Lower fitness in these growth media was significant in 5 out of 8 strains in LB and 4 out of 8 in ASM strains.

Opposite to what is observed in nutrient rich media, Cap+ strains have a strong fitness advantage in M02, M05 and AUM ( $\mathrm{P}<0.05, N=8$, one-sided t-test, difference of 1$)$. This is in agreement with our previous results that showed the maintenance of the capsule in nutrient poor media. The average fitness advantage provided by the capsule in nutrient poor media is 160 ca. 20\%. Individually, most strains had a significant fitness advantage in nutrient poor environments, with the exception of the two KL24. In these two strains, the capsule significantly imposed a fitness cost in AUM, suggesting a particular negative interaction between KL24 capsule and AUM (Figure 2).

To further corroborate the hypothesis that the amount of nutrients in the media determines the 165 fitness of capsulated strains, we competed the three strains for which the capsule was significantly costly in LB (strain \#56, \#26 and \#24, belonging to three different serotypes) in 
diluted LB (1:10). Overall, we observe a significant fitness increase of capsulated strains of $\sim 23 \%$ in the diluted rich media (Wilcoxon rank sum test, $\mathrm{P}<0.01$ for \#26 and \#24). Qualitative similar results are obtained in diluted ASM (Wilcoxon rank sum test, for strain \#24, P< 0.01). Finally, to control for potential fitness side-effects of wza deletion, due to accumulation of capsule polysaccharide in the periplasmic space (Rendueles 2020, Tan et al 2020b), we deleted either the $w b a P$ or $w c a J$ gene, the first glycosyltransferase of the capsule synthesis pathway, in the abovementioned three strains. Our results consistently show a strong fitness disadvantage of the capsule in rich media, whereas, in nutrient-poor media the

175 capsule provides a significant advantage (Figure S4B, Kruskal-Wallis, $\mathrm{P}=0.005$ ). Taken together, our results confirm that the capsule is disadvantageous in rich media, but advantageous in nutrient-poor media.

The capsule increases growth rate and population yield in nutrient poor

\section{growth media}

We investigated how the capsule could increase fitness in nutrient poor environments by assessing the effect of mutants in a number of conditions. We first tested whether the capsule provided an advantage during starvation, by increasing cell survival, as shown in

185 Streptococcus pneumoniae (Hamaguchi et al 2018). We therefore followed the survival of capsule mutants and wild type under starvation conditions. We allowed cells to starve for two months and counted surviving CFUs every month. As expected, mortality rates of Klebsiella are very low compared to other Enterobacteria (Baker et al 2019). Yet, we observed no significant differences between wild type and the capsule mutants after 30 or 60 days (Figure

190 3A and Figure S5A). To further check if there is a potential effect of the Klebsiella capsule on survival, we also challenged the strains to desiccation. No effect of the capsule on cellular survival was observed $(P>0.05, \mathrm{t}$ test for significant difference between mutants and wild type, Figure S5B).

195 Given that we observed no significant effect of the presence of the capsule on cellular survival, we tested whether differences in growth rate could account for the fitness effects observed. We thus allowed all strains and their mutants to grow in microtiter plates in all growth media and calculated the minimum generation time (Figure 3B and Figure S6A). As expected, we observed significant differences in growth rate across strains and environments 200 (GLM, $\mathrm{P}<0.001$ for both, $\mathrm{R}^{2}=0.60$ ). To assess whether the capsule affects growth dynamics 
we calculated three different parameters: minimum generation time, maximum yield and the area under the curve (AUC), which takes into account the initial population size, growth rate and carrying capacity of the environment. As expected by the competition experiments, in ASM, we observe that the capsulated strains have slower growth rate and achieve lower absorbances (ODs) a proxy for population yield (Figure 3B and Figure S6A). Interestingly, this is not the case in LB, in which strains reached on average lower yields compared to ASM. In nutrient-poor growth media, we observe the opposite to ASM, namely, that the growth rate of capsulated strains is higher and the populations reach higher yields (Figure 3B and Figure S6B). Control experiments showed a strong correlation between absorbance and cell numbers.

210 Furthermore, direct plating of cells after 16 hours of growth in microtiter plates also revealed larger population sizes in capsulated strains than in mutants in nutrient-poor environments. These observations are qualitatively similar to those observed using AUC calculations. In nutrient-poor environments, notably M02 and M005, the AUC is significantly higher in capsulated strains compared to non-capsulated strains (Figure S6C). Taken together, there is a

215 statistically significant difference in growth rate and yield between the mutants and the wild type in nutrient poor media, suggesting that the fitness benefits of capsulated strains observed in competition are due to advantages in growth dynamics. Qualitatively similar results were obtained using the three $\Delta w c a J$ and $\triangle w b a P$ mutants, namely, a higher yield and AUC of capsulated strains in nutrient poor environments but not in ASM or LB.

220

We further characterized how the capsule may affect growth to overcome the cost of its production and become an advantage in nutrient poor environments. We first tested if noncapsulated strains exhibit higher death rates in our experimental conditions. The determination of the number of dead cells after 16 hours of growth using a Live-Dead stain 225 suggests this is not so (Two-way ANOVA, $\mathrm{P}=0.47$, Figure S6D). We then hypothesized that capsulated bacteria could use their capsule as a nutrient source in nutrient poor growth media. To test this, we used the $\Delta w c a J$ and $\Delta w b a P$ mutants, to avoid capsule build up in the periplasm that could interfere with our results. We allowed the mutant strains to grow for 24 and 48 hours in minimal media with no source of carbon, in the presence of purified capsule 230 added exogenously and of uronic acid used as a standard to quantify capsule production (Figure S7). We did not observe significant differences between the treatments where the capsule was added exogenously and the respective controls (Kruskal-Wallis, P > 0.05, Figure S7). These results suggest that the advantage provided by the capsule relies in increased 
235 the capsule.

\section{Capsule mutants undergo different fates in host-mimicking media}

Our previous results show that changes in growth conditions can strongly affect selection for

the capsule. We thus sought to further understand how the capsule affected other traits that are known to be relevant during pathogenesis, and whether this effect is conserved across Klebsiella strains. We first addressed the ability of non-capsulated mutants to resist to human serum. Three Cap- mutants were significantly more sensitive to human serum than their Cap+ counterpart, whereas for the other two strains there was no significant difference (Figure 4A).

245 Resistance to human serum can also be due to the presence of different O-antigens (Doorduijn et al 2016, Merino et al 1992). In our panel, only two strains \#211 and \#26 share the same Oantigen (O1v1, Table S1). These strains have different resistance profiles, suggesting that Oantigen is not solely responsible for serum resistance. These results strongly suggest that the effect of the capsule on resistance to serum is strain-dependent (Multifactorial ANOVA, $\mathrm{P}<$ $0.001)$.

We then explored the ability of capsule mutants to survive in the presence of primary (sodium cholate - $\mathrm{CHO}-$ ) and secondary (sodium deoxycholate - $\mathrm{DCH}-$ ) bile salts, since they are antibacterial compounds that may disrupt bacterial cell membranes (Urdaneta and Casadesus

255 2017). At physiological concentrations (0.05\%), we observed no significant sensitivity of Klebsiella to bile salts (Figure S8B). When treated with 10X physiological concentrations, some, but not all, capsule mutants showed reduced viability (Figure S8).

Finally, the ability of niche colonization, specially within a host, is directly linked to the ability of the strains to adhere to surfaces and proliferate, that is, to form a biofilm. Cells within biofilms are more resilient, tolerant to antibiotics and less exposed to the host immune system (Hall-Stoodley et al 2004). We thus assessed the ability of the strains to form biofilms and determine how the capsule could impact this trait. It has been previously shown that in some strains of Klebsiella, capsule production masks the necessary fimbriae required to attach

265 to the host mucosa (Schembri et al 2004, Schembri et al 2005). On average, we do not observe a difference in the amount of biofilm between the capsulated and non-capsulated mutant forms ( $\mathrm{P}>0.05, N=9$, paired t-test, in all different growth media). However, the 
270 words, the capsule tends to contribute positively to biofilm formation in strains forming more biofilm. At the strain level, we observe that some non-capsulated mutants adhere significantly more across all environments (strain \#56) (Figure S9), whereas other strains have a stronger ability to form biofilm when the capsule is present (strain \#47) (Figure S9). This might depend on the capsule composition or it may also result from the absence of adhesion factors

275 in the strain. Overall, our data suggest that it is not the presence or absence of the capsule that affects the ability to form biofilms, but rather the amount of capsule expressed by a given strain.

Taken together, these results show that some properties like resistance to bile salts or human 280 serum, are not inherent to the presence of the capsule, but are rather strain-dependent, or dependent on the serotype-genome interaction. serotype

To further understand the interactions between the serotype, genetic background and environment, we used the original dataset of 19 strains, including at least three from each serotype. We analyzed different capsule-related variables in all five growth media. In addition to the amount of capsule production (Figure S2), the natural emergence of non-capsulated mutants (Figure 1), maximum growth rate, the maximum yield and biofilm formation, we also measured the hypermucoviscosity of strains, a trait linked to hypervirulence and driven, at least partly by the amount of capsule (Lin et al 2012) (Figures S10-S12). Firstly, we used

295 Principal Components Analysis (PCA) to analyze how the different factors (media, serotype or strain) explain the observed variance in the data. The first and second principal components explained more than $60 \%$ of the variation observed (Figure 5A). We observed no clear grouping of serotypes according to the two first axes. This suggests that the serotype is not a major determinant of the capsule-related phenotypes (Figure S13). The ellipses regrouping the

300 points by type of media show a small overlap between both rich media (ASM and LB) and these are clearly separated from the two poorest media (M005 and AUM). This suggests that most of the variance is explained by the media, presumably by the amount of nutrients 
available (Figure 5A). We then used multifactorial ANOVA to analyze quantitatively the effect of the growth media and the serotype on each trait. As expected, we observed a

305 significant effect of the growth media in all measured traits, except in biofilm formation $\left(\mathrm{R}^{2}=\right.$ $0.57, \mathrm{P}=0.06$, Table S2). As suggested by the PCA, the influence of the serotype in the capsule-associated traits is less important. Interestingly, the terms of interaction between the environment and the serotype are not statistically significant, suggesting that they are independent (Table S2).

Secondly, we sought to determine how the different capsule-related variables correlated with each other. The PCA analysis already suggested the existence of such correlations (Figure 5B). As expected, we observe a strong correlation between hypermucoviscosity (HMV) and the amount of capsule, in all individual growth media except in M005. Consistently with

315 previous reports (Lipson 2015, Pfeiffer et al 2001), we observe that the population yield is inversely proportional to the growth rate (Figure 5B). This is significant in all growth media, except M005 (for which $P=0.07$ ). Further, the amount of capsule is positively correlated with the maximum yield (Figure 5B), supporting our observation that the capsule contributes to increased yield of the population. In contrast, there is a negative correlation between the amount of capsule and the generation time, a result that is driven by growth in nutrient poor environments (Figure 5B). More precisely, in poor media, bacteria with more capsule are able to grow faster that those producing less capsule. This was not evident from the results of the analyses involving the mutant, as these showed no significant differences in generation time between the capsulated and non-capsulated strains. Overall, our results show that the capsule may impact several important cellular traits, like mucoviscosity and growth, but this is strongly dependent on the environment.

\section{DISCUSSION}

The capsule is an extracellular structure commonly associated to the ability to resist biotic or abiotic aggressions, like the immune system, phages, or desiccation (Bengoechea and $\mathrm{Sa}$ Pessoa 2019, Campos et al 2004, Paczosa and Mecsas 2016). In our previous work, we showed that capsules were more prevalent in free-living environmental bacteria than in pathogenic bacteria. This suggested that the role of the capsule in virulence may be a byproduct of adaptation outside the host, raising the question of its primary function in cells 
(Rendueles et al 2017). Here, we explore the conditions in which the capsule is advantageous despite the cost of its production and lack of antagonistic interactions and abiotic aggressions. The evolution experiments and the direct competitions show that the capsule provides a 340 disadvantage during growth in rich medium and an advantage during growth in nutrient poor environments, $c a$. $20 \%$, independently of the capsule mutants used, either outer membrane exporter $\Delta w z a$ or the initial glycosyltransferase mutants, $\Delta w c a J$ or $\Delta w b a P$.

To explain the results of our competition experiments, we examined the effect of the capsule 345 on growth dynamics of wild type strains relative to their capsule mutants. In ASM, the media allowing the highest carrying capacity, we observe that the capsule is costly since mutants grow faster and achieve higher yields. In LB, the results are more complex. Non-capsular mutants readily emerge in the evolution experiments. But there are no significant differences in terms of growth rate between $\Delta w z a$ and the wild types, as previously observed in $K$. 350 pneumoniae KPPR1, a strain not included in our panel (Mike et al 2020), and Acinetobacter baumanii (Russo et al 2010). We however observe an increased yield and a larger area under the curve in non-capsulated strains, indicative of the cost of the capsule in LB. Similarly, when using the $\Delta w c a J$ or $\Delta w b a P$ mutants, all three measures - AUC, maximum yield and growth rate- show that in LB, the capsule is costly. This might suggest that higher fitness of non-capsulated mutants in competition experiments in LB are caused by higher growth rates during exponential phase. We hypothesize that the differences in growth dynamics between $\Delta w z a$ and $\Delta w c a J$ mutants are due to the periplasmic concentration of capsule percursors (Rendueles 2020, Tan et al 2020b). Overall, we propose that due to the absence of stresses, capsule inactivation or loss in rich media leads to gains in terms of growth rate and yield, as indicated by the evolution experiments, the competitions with the $\Delta w z a$ and $\Delta w c a J$ mutants.

In nutrient poor environments, the capsule increases growth rate and populations reach higher yields. This phenomenon explains how the capsule provides a competitive advantage in nutrient poor media, despite its cost in nutrient rich media. Further, we observe a negative 365 correlation between the amount of capsule and generation time in nutrient poor environments (Figure 5B). Thus, bacteria producing more capsule are able to grow faster in nutrient poor environments. These results are somewhat counter-intuitive, as in media in which resources are scarce, sugars should be preserved for essential metabolism including energy-yielding pathways like TCA-cycle, to assemble cellular structures like the cell membrane. 
Our results challenge the current view that the primary role of the capsule is to withstand aggressions, because even during growth in pure, stable conditions, the capsule provides a significant advantage and is enough to select for its maintenance. To understand how the capsule could be advantageous for growth in nutrient poor environments, we first 375 hypothesized that capsulated strains could have reduced death rate. We did not observe any effect of the capsule in death rate during the 16 hours of our growth assay. Similarly, our twomonth long starvation experiments revealed no differences in survival between capsulated and non-capsulated strains. Previous reports had already shown that in the long term, Klebsiella spp are very resilient and have very low death rates (Bossolan et al 2005, Lappin-Scott et al 380 1988), including in lake water at temperatures close to 0 (Niemela and Vaatanen 1982) or during long-term starvation in PBS as a member of an oral community (Baker et al 2019). Alternatively, capsulated bacteria could use the capsules' polysaccharides to fuel faster growth and further multiply after consumption of all the environmental resources, as previously reported in S. pneumoniae (Hamaguchi et al 2018). However, this is inconsistent with our observation that addition of exogenous capsule to non-capsulated mutants failed to result in a significant increase of CFU in a population. Taken together, and despite of the production costs of the capsule, linked to the biosynthetic process, the energy required to polymerize and export the capsule and the use of resources that are no longer available for other cellular processes, our analysis do not provide a clear explanation on how the capsules increase population yield or growth rate. This will be the object of future research.

Since our results suggests that capsules can be advantageous in the absence of challenges, we re-assessed its impact in features known to be implicated in pathogenesis. Our results show that the impact is very variable across strains and serotypes. We observe an overall strong 395 positive correlation between the capsule and hypermucoviscosity (HMV), a proxy of hypervirulence. This would support the hypothesis that HMV is a result of capsule overproduction. However, not all strains with large capsule are HMV. This is supported by recent studies performed in the KPPR1 strain that show that capsule overproduction is not required for HMV (Mike et al 2020, Walker et al 2019). Moreover, we also show that the capsule serotype affects HMV $(\mathrm{P}=0.002)$, suggesting that the role of the capsule in HMV is convoluted, dependent on the serotype and/or the strain (Walker and Miller 2020). 
Similar to the HMV results, we observe that the role capsule in resistance to human serum is also not conserved across strains. Our results are in agreement with a previous study in which cells from different serotypes were first exposed to depolymerases, to digest the capsule (Majkowska-Skrobek et al 2018), and then tested for survival after incubation with human serum and phagocytosis. Consistently, some non-capsulated strains were more sensitive to human serum, but others seem to be as resistant as the control cultures to which no depolymerase was previously added. The diverse impact of the capsule in serum resistance is

410 also supported by a recent study which revealed that most genes contributing to serum resistance were strain-specific (Short et al 2020). This suggests that there may be strong epistasy at play between the capsule and the rest of the genome leading to full serum resistance.

415 Finally, the strains were naturally resistant to bile salts, suggesting no protective role for the capsule from bile salts at physiological levels. When bile salt concentrations are increased by a factor of ten, the capsule significantly increases survival in some strains but not all. Our results highlight that the role of capsules in traits that are relevant during pathogenesis are diverse and may depend on the strains, the environment, the serotype, and genetic interactions

420 with the background. This implies that the role of capsules in pathogenesis varies across Klebsiella. This is supported by the fact that some serotypes are associated to hypervirulence, like $\mathrm{K} 1$ and $\mathrm{K} 2$, whereas other serotypes are less virulent but associated to multi-drug resistant like KL107 (Wyres et al 2019). In an experiment in which the capsule locus was exchanged between strains with a K2 and K21a serotype, it was shown that the capacity to escape from macrophages, and thus virulence, was inherited with the capsule serotype (Ofek et al 1993). The fact that the roles of the capsule during pathogenesis are not conserved also supports the notion that the benefits of the capsule for virulence are a by-product of adaptation in non-host related contexts. Accordingly, a recent study showed that carbapenemresistant $K$. pneumoniae ST258 unencapsulated isolates formed more biofilm and could 430 invade the epithelial bladder tissue more effectively than capsulated isolates. This resulted in the formation of intracellular infective reservoirs that lead to recurrent urinary infections (Ernst et al 2020). Overall, our analyses of multiple strains with different serotypes revealed that the role of capsule in traits that may be relevant during pathogenesis is complex and depends not only on the strain but also the serotype. 
To conclude, the complex relation between the capsule and the host genome highlights that there are yet many research venues to be explored in terms of selection forces acting on the maintenance and diversification of the capsule, its function across a diversity of strains, and potential epistasy between the capsule and the rest of the genome. Here, we show that the 440 growth media affects the relative fitness of capsulated bacteria, and alone justifies the maintenance of the capsule in a population. Most literature suggests that the rapid turnover of capsular serotypes is due to biotic pressures, such as phage predation, cell-to-cell interactions, the host adaptive immune system, or protozoa grazing (Cobey and Lipsitch 2012, Mostowy and Holt 2018), but more work is needed to elucidate whether the abiotic conditions of the

445 environment also play a role in the diversification of the capsule composition. For example, different capsules could be better adapted to certain poor environments because of their different propensity to aggregate or because of their organization at the cell surface (Phanphak et al 2019, Rendueles 2020). It could also be that different serotypes are associated to specific functions or fitness advantages (Ofek et al 1993). This might lead to selection of serotype switching when bacteria colonize novel environments. Finally, more work is needed to elucidate capsule functions other than those that are commonly evoked and provide more insights into the non-universality of the role of capsules both within and across species (Rendueles 2020). Indeed, recent studies show that the capsule does not protect against temperate or virulent phages in $K$. pneumoniae, but rather the opposite, the capsule acts as a receptor for phages (de Sousa et al 2020, Tan et al 2020a). Taken together, our work suggests that the primary evolutionary forces selecting for the existence of a capsule may be very different from those selecting for its subsequent diversification, and highlights that much work is still needed to fully understand the primary role of capsules, both at the molecular and at the ecological level. 


\section{MATERIALS AND METHODS}

Bacterial strains and growth conditions. Bacterial strains and plasmids used in this study are listed in Table S1. Primers used for the construction of mutants are listed in Table S3. Bacteria were grown at $37^{\circ}$ in Luria-Bertani (LB) agar plates or in $4 \mathrm{~mL}$ of liquid broth under

465 vigorous shaking $(250 \mathrm{rpm})$ unless indicated otherwise. Kanamycin $(50 \mu \mathrm{g} / \mathrm{ml})$ or Streptomycin $(100 \mu \mathrm{g} / \mathrm{mL}$ for E. coli and $200 \mu \mathrm{g} / \mathrm{ml}$ for Klebsiella $)$ were used in the selection of the plasmids strain selection and cultures. Antibiotics were purchased from Sigma.

Growth media description. AUM and ASM stand for artificial urine medium and artificial sputum medium, respectively, and were done as described previously (Brooks and Keevil 470 1997, Fung et al 2010). Briefly, AUM is mainly composed of urea and peptone with trace amounts of lactic acid, uric acid, creatinine and peptone. ASM is composed of mucin, DNA, egg yolk and aminoacids. LB is composed of tryptone and yeast extract. M02 and M005 indicate minimal M63B1 supplemented with $0.2 \%$ and $0.05 \%$ glucose (sole carbon source), respectively.

475 Determination of serotype. Complete or draft genomes were serotyped using Kaptive with default settings (Wick et al 2018).

Natural loss of capsule. An overnight culture in LB was diluted at 1:100 in the different environments (ASM, LB, M02, M005, AUM) in a final volume of $4 \mathrm{~mL}$. Cultures were allowed to grow for 24 hours at $37^{\circ} \mathrm{C}$ and diluted again to $1: 100$ in fresh media. This was repeated for 3 times. Then, each culture in each environment was serially diluted and CFUs were counted ( 3 plates per sample, > 100 colonies per plate). Non-capsulated mutants are easily visualized by the naked eye as mutants produce smaller, rough and translucent colonies. Results are expressed as a ratio of non-capsulated mutant colonies observed over the total plate count. The limit of detection of non-capsulated mutants in this assay was $0.22 \% \pm$ 4850.04 or 1:450 per biological replicate per environment. To ensure that the naturally-emerged non-capsulated colonies observed on plate are bona fide genetic mutants and not phasevariants, we randomly chose 10 populations, using the sample function in R. Three noncapsulated clones from each population were randomly selected and restreaked twice. All clones derived from the original non-capsulated colony remained non-capsulated, demonstrating that the loss of the capsule is genetic. 
Generation of $\Delta w z a, \Delta w c a J$ and $\Delta w b a P$ capsule mutants. Isogenic capsule mutants were constructed by an in-frame deletion of $w z a$ by allelic exchange. Upstream and downstream sequences of each gene (> 500pb) were amplified using Phusion Master Mix (Thermo 495 Scientific) then joined by overlap PCR. The resulting PCR product was purified using the QIAquick Gel Extraction Kit and then cloned with the Zero Blunt ${ }^{\circledR}$ TOPO ${ }^{\circledR}$ PCR Cloning Kit (Invitrogen) into competent $E$. coli $\mathrm{DH} 5 \alpha$ strain. $\mathrm{KmR}$ colonies were isolated and checked by PCR. Cloned Zero Blunt ${ }^{\circledR}$ TOPO $^{\circledR}$ plasmid was extracted using the QIAprep Spin Miniprep $\mathrm{Kit}$, and digested for 2 hours at $37^{\circ} \mathrm{C}$ with ApaI and SpeI restriction enzymes and ligated with

500 T4 DNA ligase (Promega) overnight at $16^{\circ}$ to a double-digested pKNG101 plasmid. The ligation was transformed into competent E. coli $\mathrm{DH} 5 \alpha$ pir strain, and again into E. coli $\mathrm{MFD}$ $\lambda$-pir strain (Ferrieres et al 2010), used as a donor strain for conjugation into Klebsiella spp. Conjugations were performed for 24 hours at $37^{\circ} \mathrm{C}$. Single cross-over mutants (transconjugants) were selected on Streptomycin plates $(200 \mu \mathrm{g} / \mathrm{ml})$. To select for plasmid excision, transconjugants were grown for 16 hours at $25^{\circ} \mathrm{C}$ and double cross-over mutants were selected on LB without salt and supplemented with 5\% sucrose at room temperature. To confirm the loss of the plasmid, colonies were tested for their sensitivity to streptomycin. From each double-recombination, a mutant and a wild type were isolated. All experiments were performed in parallel with the parental strain, deletion mutant and the associated wild 510 type, to ensure that the phenotype observed was directly due to the deletion and not to other potential SNPs that could have accumulated in the genome during the mutant generation, and thus present in both the mutant and the wild type.

Deletion mutants were first verified by Sanger, and then checked for off-target mutations using Illumina. $\Delta w b a P$ and $\Delta w c a J$ did not present any off-target mutations. Four $\Delta w z a$ strains

515 presented off-target mutations. Strain \#63 (KL107) $\Delta w z a$ has a 15bp deletion upstream of $r f a H$, a major capsule regulator. Inactivation of $r f a H$ inhibits the initiation of capsule synthesis altogether. Strain \#37 (KL24) has an extra 15 bp deletion just before the ATG of $w z a$. Strain \#51 (KL24) has a SNP in pcaR. There are no reports on a direct influence of this gene on capsule production. Strain \#55 (KL2) has a SNP in sacX gene which results in

520 delayed growth. Despite the fact that these strains are bona fide capsule mutants, as shown by microscopic observation and capsule quantification, the results are presented as supplemental figures. 
Microscopy. Bacteria capsules were visualized using Indian Ink coloration on an exponential

525 phase culture. Images were acquired on a Nikon Eclipse E400 camera using the Digital Net DN100 camera control unit and a 100X objective.

Capsule extraction and quantification. The bacterial capsule was extracted as described in (Domenico et al 1989) and quantified by using the uronic acid method (Blumenkrantz and 530 Asboe-Hansen 1973). Briefly, $500 \mu \mathrm{L}$ of an overnight culture was mixed with $100 \mu \mathrm{L}$ of $1 \%$ Zwittergent 3-14 detergent in $100 \mathrm{mM}$ citric acid ( $\mathrm{pH} 2.0)$ and heated at $56^{\circ} \mathrm{C}$ for 20 minutes. After it was centrifuged for $5 \mathrm{~min}$ at $14,000 \mathrm{rpm}$ and $300 \mu \mathrm{L}$ of the supernatant was transferred to a new tube. Absolute ethanol was added to a final concentration of $80 \%$ and the tubes were placed on ice for 20 minutes. After a second wash with ethanol at $70 \%$, the pellet was dried and dissolved in $250 \mu \mathrm{L}$ of distilled water. The pellet was then incubated for 2 hours at $56^{\circ} \mathrm{C}$. Polysaccharides were then quantified by measuring the amount of uronic acid. A $1,200 \mu \mathrm{L}$ volume of $0.0125 \mathrm{M}$ tetraborate in concentrated $\mathrm{H}_{2} \mathrm{SO}_{4}$ was added to $200 \mu \mathrm{L}$ of the sample to be tested. The mixture was vigorously vortexed and heated in a boiling-water bath for $5 \mathrm{~min}$. The mixture was allowed to cool, and $20 \mu \mathrm{L}$ of $0.15 \%$ 3-hydroxydiphenol in

$540 \quad 0.5 \% \mathrm{NaOH}$ was added. The tubes were shaken, and $100 \mu \mathrm{L}$ were transferred to a microtiter plate for absorbance measurements $(520 \mathrm{~nm})$. The uronic acid concentration in each sample was determined from a standard curve of glucuronic acid.

Competition assay. Mutants and their respective wild types were grown overnight in LB.

545 They were then mixed in a 1:1 proportion. A sample was taken and used for serial dilution and CFU counting as a control of $\mathrm{T}_{0}$. The co-culture was then diluted $1 / 100$ in $4 \mathrm{~mL}$ of the different environments (i.e. LB, AUM, ASM, M02 and M005). For control experiments, ASM and LB were diluted 1:10 in M63B1 with no supplement of glucose. After 24 hours of competition $\left(\mathrm{T}_{24}\right)$, each mixture was serially diluted and plated. Capsulated and non-

550 capsulated colonies are clearly differentiated visually and counted separately. The competitive index of capsulated strains was calculated by dividing the ratio of CFU at $\mathrm{T}_{24}$ over $\mathrm{T}_{0}$.

Growth curves. Overnight cultures were diluted at 1:100 in the aforementioned 555 environments. $200 \mu \mathrm{l}$ of each subculture was transferred in a 96-well microplate. Cell densities of cultures were estimated with a TECAN Genios ${ }^{\mathrm{TM}}$ plate reader. Absorbance 
$\left(\mathrm{OD}_{600}\right)$. Light absorbance values of each well reflect the average of 6 reads per well. Absorbance values from within-block technical replicates were averaged and these averages were used as statistically independent data points. (i) Growth rate. Minimum generation times were estimated across replicates for the 1 hour interval $(\Delta \mathrm{T})$ spanning the fastest growth during exponential growth phase. This was calculated as:

$$
\frac{1}{T d}=\frac{\log _{10}\left(\text { maximum }_{\mathrm{OD} 600} / \text { minimum }_{\mathrm{OD} 600}\right)}{\log _{10}(2) * \Delta T}
$$

(ii) Maximum yield. Relative yields were calculated measuring ratios of the maximum absorbance of the assay compared to the reference (parental strain). The experiments were

565 performed at least three independent times, with three technical replicates per experiment. The average of the three technical replicates per experiment was used for statistical purposes. Control experiments were performed to assess the correlation between $\mathrm{CFU}$ and $\mathrm{OD}_{600}$ during growth for both capsulated (Spearman's rho $=1.0, \mathrm{P}=0.002$ ) and non-capsulated mutants (Spearman's rho $=0.94, \mathrm{P}=0.016$ ). To confirm the differences in yield observed in minimal 570 medium between capsulated and non-capsulated strains, we performed a control experiment $(\mathrm{N}=3)$ in which we grew the strains \#56 and \#24 in microtiterplates as described before. After 16h, we plated an aliquot to count CFUs. Significantly less CFUs were observed in the non-capsulated mutants compared to wild type ( $\mathrm{P}<0.05$ for both strains). (iii) Area under the curve (AUC). AUC was calculated using the R function trapz from the pracma package.

Serum resistance. The ability of Klebsiella strains to resist killing by serum was performed as described previously (Dorman et al 2018, Podschun et al 1993, Podschun and Ullmann 1998). Briefly, bacteria were grown in LB to OD $600 \mathrm{~nm}$ of 1, pelleted by centrifugation and resuspended in sterile PBS 1X. $200 \mu \mathrm{l}$ of bacterial suspension was added to $400 \mu \mathrm{l}$ of pre580 warmed at $37^{\circ} \mathrm{C}$ human sera (Sigma-Aldrich S7023), and the mixture was incubated for $2 \mathrm{~h}$ at $37^{\circ} \mathrm{C}$. Control reactions were performed with serum inactivated by heat at $56^{\circ} \mathrm{C}$ for $30 \mathrm{~min}$. Reactions were stopped by placing on ice, and viable bacterial counts were determined before and after incubation by serial dilution and CFU plating.

585 Bile salts resistance. Bacteria were grown overnight in LB. As previously described (Tan et al 2020b), cultures were then serially diluted and plated in LB supplemented with either 0, $0.05 \%$ (physiological conditions) and $0.5 \%$ of $\mathrm{CHO}$ (primary bile salt, sodium cholate) or DCH (secondary bile salt, sodium deoxycholate). Colonies were allowed to grow and 
590 experiment was used for statistical purposes.

Starvation. Bacteria were grown in $\mathrm{LB}$ to $\mathrm{OD}=0.2,1 \mathrm{~mL}$ of the culture was pelleted by centrifugation and, resuspended in $400 \mu \mathrm{l}$ of PBS 1X, as in (Hamaguchi et al 2018). Cultures were allowed to starve at room temperature. Aliquots were taken at 0,30 and 60 days, and used for serial dilutions and CFU plating.

Biofilm Production. The ability of strains to form biofilm was performed as described in (Rendueles et al 2011). Overnight cultures were diluted at 1:100 in the different growth media mentioned earlier. $100 \mu \mathrm{L}$ of each diluted culture was transferred in triplicate into 96-well microplate wells and allowed to grow for 24 hours without shaking at $37^{\circ} \mathrm{C}$. Cells were gently 600 removed from the wells and washed once in distilled water. To stain biofilms, $125 \mu \mathrm{L}$ of $1 \%$ crystal violet was added to each well for 20 minutes. The crystal violet was gently decanted and each well was washed three times with distilled water. After the plate was totally dry, the biofilm was solubilized for $10 \mathrm{~min}$ in $150 \mu \mathrm{L}$ of mix with $80 \%$ ethanol and $20 \%$ acetone. The absorbance of the sample was read at $\mathrm{OD}_{570}$. At least 3 biological independent replicates were 605 performed for each sample.

Hypermucoviscosity. The optical density $\mathrm{OD}_{600}$ of overnight cultures were first determined by spectrophotometry as described in (Dorman et al 2018). The cultures were then sedimented by slow centrifugation (at 2,500 X g) for $5 \mathrm{~min}$ at room temperature. The optical density $\mathrm{OD}_{600}$ of the top $650 \mu \mathrm{L}$ of supernatant was determined. Results were expressed as a ratio of 610 the supernatant $\mathrm{OD}_{600}$ to that of the input culture.

Dessication test. Dessication assay was carried as previously described in (Chin et al 2018). Briefly, $25 \mu \mathrm{L}$ of exponentially-growing Klebsiella strains were deposited at the bottom of microtiterplates, and allowed the culture to dry under a laminar flow hood. Strains were then left either at $37^{\circ}$ for one, two or four days or at room temperature for two, four, seven and 14 days. PBS was added to resuspend the cultures and diluted serially from CFU counts.

Quantification of dead cells. Cells were allowed to grow as described above with a minor modification; over day cultures were used to inoculate the 96-well microtiter plates. After 16 hours of growth in M02, dead cells were quantified using the LIVE/DEAD ${ }^{\mathrm{TM}}$ BacLight $^{\mathrm{TM}}$ Bacterial Viability Kit from Invitrogen, following provider instructions. Images were 
620 acquired on a Nikon Eclipse E400 microscope using the Digital Net DN100 camera control unit and a 100X objective. Cells were counted using ImageJ software (https://imagej.net/). On average, 6 pictures were taken per sample $(\min =3, \max =10)$ and $c a$. 550 cells per sample were observed $(\min =161, \max =1604)$. The experiment was performed four times.

625 Growth on exogenously added capsule. Capsules were extracted from either wild type strains or their respective $\Delta w c a J$ or $\Delta w b a P$ (for strain \#24) mutants and quantified as described above. To initiate growth, overnight cultures of $\Delta w c a J$ mutants were diluted at 1:100 in M63B1 media. Media was supplemented with either $0.8 \mathrm{ng} / \mu \mathrm{L}$ of extracted capsule from wild type or an equivalent volume extracted from the $\Delta w c a J$ or $\Delta w b a P$ (for strain 24)

630 mutants. As a negative control, cultures were allowed to grow in M63B1 with no nutrients. In parallel, M63B1 was supplemented with glucuronic acid (the standard used for estimating capsule concentration) at $0.8 \mathrm{ng} / \mu \mathrm{L}$ final. Growth was allowed for 48 hours at $37^{\circ}$ and 250 rpm. Bacterial counts were performed at $\mathrm{T}_{0}, \mathrm{~T}_{24}$ and $\mathrm{T}_{48}$ by serial dilution and plating. Results are expressed as the increase in CFU over $24 \mathrm{~h}$.

\section{REFERENCES}

Bagley ST (1985). Habitat association of Klebsiella species. Infect Control 6: 52-58.

Baker JL, Hendrickson EL, Tang XY, Lux R, He XS, Edlund A et al (2019). Klebsiella and 640 Providencia emerge as lone survivors following long-term starvation of oral microbiota. Proc Natl Acad Sci USA 116: 8499-8504.

Band VI, Weiss DS (2015). Mechanisms of Antimicrobial Peptide Resistance in GramNegative Bacteria. Antibiotics (Basel) 4: 18-41.

Bengoechea JA, Pessoa JS (2019). Klebsiella pneumoniae infection biology: living to counteract host defences. Fems Microbiology Reviews 43: 123-144.

Bengoechea JA, Sa Pessoa J (2019). Klebsiella pneumoniae infection biology: living to counteract host defences. FEMS Microbiol Rev 43: 123-144.

Bentley SD, Aanensen DM, Mavroidi A, Saunders D, Rabbinowitsch E, Collins M et al (2006). Genetic analysis of the capsular biosynthetic locus from all 90 pneumococcal serotypes. PLoS Genet 2: e31.

Blin C, Passet V, Touchon M, Rocha EPC, Brisse S (2017). Metabolic diversity of the emerging pathogenic lineages of Klebsiella pneumoniae. Environ Microbiol 19: 1881-1898.

Blumenkrantz N, Asboe-Hansen G (1973). New method for quantitative determination of 
Bossolan N, Godinho MJL, Volpon AGT (2005). Growth and starvation of a strain of Klebsiella pneumoniae isolated from a Brazilian oil formation. World J Microb Biot 21: 1471-1475.

665

Brooks T, Keevil CW (1997). A simple artificial urine for the growth of urinary pathogens. Lett Appl Microbiol 24: 203-206.

Campos MA, Vargas MA, Regueiro V, Llompart CM, Alberti S, Bengoechea JA (2004).

670 Capsule polysaccharide mediates bacterial resistance to antimicrobial peptides. Infect Immun 72: 7107-7114.

Caneiras C, Lito L, Melo-Cristino J, Duarte A (2019). Community- and Hospital-Acquired Klebsiella pneumoniae Urinary Tract Infections in Portugal: Virulence and Antibiotic

675 Resistance. Microorganisms 7: 138.

Chin CY, Tipton KA, Farokhyfar M, Burd EM, Weiss DS, Rather PN (2018). A highfrequency phenotypic switch links bacterial virulence and environmental survival in Acinetobacter baumannii. Nat Microbiol 3: 563-569.

680

Cobey S, Lipsitch M (2012). Niche and neutral effects of acquired immunity permit coexistence of pneumococcal serotypes. Science 335: 1376-1380.

Croucher NJ, Finkelstein JA, Pelton SI, Mitchell PK, Lee GM, Parkhill J et al (2013).

685 Population genomics of post-vaccine changes in pneumococcal epidemiology. Nat Genet 45: 656-663.

Croucher NJ, Kagedan L, Thompson CM, Parkhill J, Bentley SD, Finkelstein JA et al (2015). Selective and genetic constraints on pneumococcal serotype switching. PLoS Genet 11:

690 e1005095.

de Sousa JAM, Buffet A, Haudiquet M, Rocha EPC, Rendueles O (2020). Modular prophage interactions driven by capsule serotype select for capsule loss under phage predation. ISME J, in press

Domenico P, Schwartz S, Cunha BA (1989). Reduction of capsular polysaccharide production in Klebsiella pneumoniae by sodium salicylate. Infect Immun 57: 3778-3782.

Doorduijn DJ, Rooijakkers SH, van Schaik W, Bardoel BW (2016). Complement resistance 700 mechanisms of Klebsiella pneumoniae. Immunobiology 221: 1102-1109.

Dorman MJ, Feltwell T, Goulding DA, Parkhill J, Short FL (2018). The Capsule Regulatory Network of Klebsiella pneumoniae Defined by density-TraDISort. MBio 9: e01863-01818.

705 Ernst CM, Braxton JR, Rodriguez-Osorio CA, Zagieboylo AP, Li L, Pironti A et al (2020). Adaptive evolution of virulence and persistence in carbapenem-resistant Klebsiella pneumoniae. Nat Med 26: 705-711. 
710 expression in Streptococcus pneumoniae negatively affects spontaneous and antibioticinduced lysis and contributes to antibiotic tolerance. J Infect Dis 189: 328-338.

Ferrieres L, Hemery G, Nham T, Guerout AM, Mazel D, Beloin C et al (2010). Silent mischief: bacteriophage $\mathrm{Mu}$ insertions contaminate products of Escherichia coli random

715 mutagenesis performed using suicidal transposon delivery plasmids mobilized by broad-hostrange RP4 conjugative machinery. J Bacteriol 192: 6418-6427.

Follador R, Heinz E, Wyres KL, Ellington MJ, Kowarik M, Holt KE et al (2016). The diversity of Klebsiella pneumoniae surface polysaccharides. Microb Genom 2: e000073.

Fung C, Naughton S, Turnbull L, Tingpej P, Rose B, Arthur J et al (2010). Gene expression of Pseudomonas aeruginosa in a mucin-containing synthetic growth medium mimicking cystic fibrosis lung sputum. J Med Microbiol 59: 1089-1100.

725 Geisinger E, Huo W, Hernandez-Bird J, Isberg RR (2019). Acinetobacter baumannii: Envelope Determinants That Control Drug Resistance, Virulence, and Surface Variability. Annu Rev Microbiol 73: 481-506.

Giguere D (2015). Surface polysaccharides from Acinetobacter baumannii: Structures and 730 syntheses. Carbohydrate research 418: 29-43.

Guerry P, Poly F, Riddle M, Maue AC, Chen YH, Monteiro MA (2012). Campylobacter polysaccharide capsules: virulence and vaccines. Front Cell Infect Microbiol 2: 7.

735 Hall-Stoodley L, Costerton JW, Stoodley P (2004). Bacterial biofilms: from the natural environment to infectious diseases. Nat Rev Microbiol 2: 95-108.

Hamaguchi S, Zafar MA, Cammer M, Weiser JN (2018). Capsule Prolongs Survival of Streptococcus pneumoniae during Starvation. Infect Immun 86: e00802-00817.

Julianelle LA (1928). Bacterial Variation in Cultures of Friedlander's Bacillus. J Exp Med 47: 889-902.

Jung SY, Matin A, Kim KS, Khan NA (2007). The capsule plays an important role in 745 Escherichia coli K1 interactions with Acanthamoeba. Int J Parasitol 37: 417-423.

Lamb KE, Flasche S, Diggle M, Inverarity D, Greenhalgh D, Jefferies JM et al (2014). Trends in serotypes and sequence types among cases of invasive pneumococcal disease in Scotland, 1999-2010. Vaccine 32: 4356-4363.

Lappin-Scott HM, Cusack F, MacLeod A, Costerton JW (1988). Starvation and nutrient resuscitation of Klebsiella pneumoniae isolated from oil well waters. J Appl Bacteriol 64: 541-549.

755 Lee CR, Lee JH, Park KS, Jeon JH, Kim YB, Cha CJ et al (2017). Antimicrobial Resistance of Hypervirulent Klebsiella pneumoniae: Epidemiology, Hypervirulence-Associated Determinants, and Resistance Mechanisms. Front Cell Infect Microbiol 7: 483. 
Levins R (1968). Evolution in changing environments; some theoretical explorations. Princeton University Press: Princeton, NJ.

Lin TL, Yang FL, Yang AS, Peng HP, Li TL, Tsai MD et al (2012). Amino acid substitutions of MagA in Klebsiella pneumoniae affect the biosynthesis of the capsular polysaccharide. Plos One 7: e46783.

Lipson DA (2015). The complex relationship between microbial growth rate and yield and its implications for ecosystem processes. Frontiers in Microbiology 6: 615.

Majkowska-Skrobek G, Latka A, Berisio R, Squeglia F, Maciejewska B, Briers Y et al 770 (2018). Phage-Borne Depolymerases Decrease Klebsiella pneumoniae Resistance to Innate Defense Mechanisms. Front Microbiol 9: 2517.

McBride SM, Fischetti VA, LeBlanc DJ, Moellering RC, Gilmore MS (2007). Genetic Diversity among Enterococcus faecalis. Plos One 2: e582.

Merino S, Camprubi S, Alberti S, Benedi VJ, Tomas JM (1992). Mechanisms of Klebsiella pneumoniae resistance to complement-mediated killing. Infect Immun 60: 2529-2535.

Meyers LA, Bull JJ (2002). Fighting change with change: adaptive variation in an uncertain world. Trends Ecol Evol 17: 551-557.

Mike LA, Stark AJ, Forsyth VS, Vornhagen J, Smith SN, Bachman MA et al (2020). A systematic analysis of hypermucoviscosity and capsule reveals distinct and overlapping genes that impact Klebsiella pneumoniae fitness. bioRxiv: doi.org/10.1101/2020.06.22.164582

Mori M, Ohta M, Agata N, Kido N, Arakawa Y, Ito H et al (1989). Identification of species and capsular types of Klebsiella clinical isolates, with special reference to Klebsiella planticola. Microbiol Immunol 33: 887-895.

Mostowy RJ, Croucher NJ, De Maio N, Chewapreecha C, Salter SJ, Turner P et al (2017). Pneumococcal Capsule Synthesis Locus cps as Evolutionary Hotspot with Potential to Generate Novel Serotypes by Recombination. Mol Biol Evol 34: 2537-2554.

795 Mostowy RJ, Holt KE (2018). Diversity-Generating Machines: Genetics of Bacterial SugarCoating. Trends Microbiol 26: 1008-1021.

Niemela SI, Vaatanen P (1982). Survival in lake water of Klebsiella pneumoniae discharged by a paper mill. Appl Environ Microbiol 44: 264-269.

800

Ofek I, Kabha K, Athamna A, Frankel G, Wozniak DJ, HAsty DL et al (1993). Genetic Exchange of Determinants for Capsular Polysaccharide Biosynthesis between Klebsiella pneumoniae Strains Expressing Serotypes K2 and K2la. Infect Immun 61: 4208-4216.

805 Ophir T, Gutnick DL (1994). A role for exopolysaccharides in the protection of microorganisms from desiccation. Appl Environ Microbiol 60: 740-745. 
Orskov I (1955). Serological investigations in the Klebsiella group. I. New capsule types. Acta Pathol Microbiol Scand 36: 449-453.

810

Paczosa MK, Mecsas J (2016). Klebsiella pneumoniae: Going on the Offense with a Strong Defense. Microbiol Mol Biol Rev 80: 629-661.

Pfeiffer T, Schuster S, Bonhoeffer S (2001). Cooperation and competition in the evolution of 815 ATP-producing pathways. Science 292: 504-507.

Phanphak S, Georgiades P, Li R, King J, Roberts IS, Waigh TA (2019). Super-Resolution Fluorescence Microscopy Study of the Production of K1 Capsules by Escherichia coli: Evidence for the Differential Distribution of the Capsule at the Poles and the Equator of the 820 Cell. Langmuir 35: 5635-5646.

Podschun R, Ullmann U (1992). Klebsiella capsular type K7 in relation to toxicity, susceptibility to phagocytosis and resistance to serum. J Med Microbiol 36: 250-254.

825 Podschun R, Sievers D, Fischer A, Ullmann U (1993). Serotypes, hemagglutinins, siderophore synthesis, and serum resistance of Klebsiella isolates causing human urinary tract infections. J Infect Dis 168: 1415-1421.

Podschun R, Ullmann U (1998). Klebsiella spp. as nosocomial pathogens: Epidemiology,

830 taxonomy, typing methods, and pathogenicity factors. Clinical Microbiol Rev 11: 589-603.

Randall WA (1939). Colony and Antigenic Variation in Klebsiella pneumoniae Types A, B and C. J Bacteriol 38: 461-477.

835 Rendueles O, Travier L, Latour-Lambert P, Fontaine T, Magnus J, Denamur E et al (2011). Screening of Escherichia coli species biodiversity reveals new biofilm-associated antiadhesion polysaccharides. MBio 2: e00043-00011.

Rendueles O, Garcia-Garcera M, Neron B, Touchon M, Rocha EPC (2017). Abundance and 840 co-occurrence of extracellular capsules increase environmental breadth: Implications for the emergence of pathogens. PLoS Pathog 13: e1006525.

Rendueles O (2020). Deciphering the role of the capsule of Klebsiella pneumoniae during pathogenesis: A cautionary tale. Mol Microbiol 113: 883-888.

Rittershaus ES, Baek SH, Sassetti CM (2013). The normalcy of dormancy: common themes in microbial quiescence. Cell Host Microbe 13: 643-651.

850 Russo TA, Luke NR, Beanan JM, Olson R, Sauberan SL, MacDonald U et al (2010). The K1 capsular polysaccharide of Acinetobacter baumannii strain 307-0294 is a major virulence factor. Infect Immun 78: 3993-4000.

Schembri MA, Dalsgaard D, Klemm P (2004). Capsule shields the function of short bacterial adhesins. J Bacteriol 186: 1249-1257. 
Schembri MA, Blom J, Krogfelt KA, Klemm P (2005). Capsule and fimbria interaction in Klebsiella pneumoniae. Infect Immun 73: 4626-4633.

860 Short FL, Di Sario G, Reichmann NT, Kleanthous C, Parkhill J, Taylor PW (2020). Genomic profiling reveals distinct routes to complement resistance in Klebsiella pneumoniae. Infect Immun 88: e00043-00020.

Tan D, Zhang Y, Qin J, Le S, Gu J, Chen LK et al (2020a). A Frameshift Mutation in wcaJ

865 Associated with Phage Resistance in Klebsiella pneumoniae. Microorganisms 8: 378.

Tan YH, Chen Y, Chu W, Sham L, Gan YH (2020b). Cell envelope defects of different capsule-null mutants in K1 hypervirulent Klebsiella pneumoniae can affect bacterial pathogenesis. Mol Microbiol 113: 889-905.

Thrane SW, Taylor VL, Freschi L, Kukavica-Ibrulj I, Boyle B, Laroche J et al (2015). The Widespread Multidrug-Resistant Serotype O12 Pseudomonas aeruginosa Clone Emerged through Concomitant Horizontal Transfer of Serotype Antigen and Antibiotic Resistance Gene Clusters. MBio 6: e01396-01315.

Tipton KA, Chin CY, Farokhyfar M, Weiss DS, Rather PN (2018). Role of Capsule in Resistance to Disinfectants, Host Antimicrobials, and Desiccation in Acinetobacter baumannii. Antimicrob Agents Chemother 62: e01188-01118.

880 Tomas JM, Benedi VJ, Ciurana B, Jofre J (1986). Role of capsule and O antigen in resistance of Klebsiella pneumoniae to serum bactericidal activity. Infect Immun 54: 85-89.

Urdaneta V, Casadesus J (2017). Interactions between Bacteria and Bile Salts in the Gastrointestinal and Hepatobiliary Tracts. Front Med-Lausanne 4: 163.

Wacharotayankun R, Arakawa Y, Ohta M, Hasegawa T, Mori M, Horii T et al (1992). Involvement of rcsB in Klebsiella K2 capsule synthesis in Escherichia coli K-12. J Bacteriol 174: $1063-1067$.

890 Walker KA, Miner TA, Palacios M, Trzilova D, Frederick DR, Broberg CA et al (2019). A Klebsiella pneumoniae Regulatory Mutant Has Reduced Capsule Expression but Retains Hypermucoviscosity. mBio 10: e00089-00019.

Walker KA, Miller VL (2020). The intersection of capsule gene expression,

895 hypermucoviscosity and hypervirulence in Klebsiella pneumoniae. Curr Opin Microbiol 54: 95-102.

Wick RR, Heinz E, Holt KE, Wyres KL (2018). Kaptive Web: User-Friendly Capsule and Lipopolysaccharide Serotype Prediction for Klebsiella Genomes. J Clin Microbiol 56 e00197-18.

Williams P, Lambert PA, Brown MR, Jones RJ (1983). The role of the $\mathrm{O}$ and $\mathrm{K}$ antigens in determining the resistance of Klebsiella aerogenes to serum killing and phagocytosis. J Gen Microbiol 129: 2181-2191. 
Wyres KL, Gorrie C, Edwards DJ, Wertheim HFL, Hsu LY, Van Kinh N et al (2015). Extensive Capsule Locus Variation and Large-Scale Genomic Recombination within the Klebsiella pneumoniae Clonal Group 258. Genome Biology and Evolution 7: 1267-1279.

910 Wyres KL, Wick RR, Gorrie C, Jenney A, Follador R, Thomson NR et al (2016). Identification of Klebsiella capsule synthesis loci from whole genome data. Microb Genom 2: e000102.

Wyres KL, Wick RR, Judd LM, Froumine R, Tokolyi A, Gorrie CL et al (2019). Distinct

915 evolutionary dynamics of horizontal gene transfer in drug resistant and virulent clones of Klebsiella pneumoniae. PLoS Genet 15: e1008114.

Wyres KL, Lam MMC, Holt KE (2020). Population genomics of Klebsiella pneumoniae. Nat Rev Microbiol 18: 344-359.

\section{ACKNOWLEDGEMENTS}

We thank Matthieu Haudiquet, Jean-Marc Ghigo and Nienke Buddelmeijer for critical reading of the manuscript. We thank Sylvain Brisse for providing us with the necessary 925 Klebsiella strains, and Christiane Forestier and Damien Balestrino for providing the pKNG101 plasmid used to generate the mutants. We would also like to thank Jean-Marc Ghigo and Christophe Beloin for the gift of E. coli S17 MFD $\lambda$-pir strain and E. coli DH5 $\alpha$ pir.

\section{FUNDING}

This work was supported by an ANR JCJC (Agence national de recherche) grant [ANR 18 CE12 000101 ENCAPSULATION] awarded to O.R.. The laboratory is funded by a Laboratoire d'Excellence 'Integrative Biology of Emerging Infectious Diseases' grant [ANR10-LABX-62-IBEID], the INCEPTION programme [PIA/ANR-16-CONV-0005], and the 935 FRM [EQU201903007835]. The funders had no role in the study design, data collection and interpretation, or the decision to submit the work for publication. 


\section{FIGURE LEGENDS}

940 Figure 1. Frequency of capsulated colonies across strains and growth media after three days of evolution. Strains were diluted 1:100 and inoculated in fresh media every 24 hours during three days. Strain \#212 was unable to grow in M005. Points represent the average loss of capsule across at least three independently evolving populations per strain. Error bars represent standard deviation across replicate populations.

Figure 2. Competition between capsule mutants and their associated wild type in different growth media. Each point represents the average of at least three independent replicates. Individual error bars for each strain are not displayed for clarity purposes. Colours represent different serotypes (as in Figure 1), whereas the shape represent different species. Results for the strains with no compensatory mutations are presented in the supplemental material (Figure S4). $* \mathrm{P}<0.05 ; * * \mathrm{P}<0.01$, Tukey post hoc and correction for multiple tests.

Figure 3. Survival, growth rate and yield of strains in each environment. A. Survival of parental, $\Delta w z a$ mutant and the associated wild type, relative to day 0. Data is $\log _{10}$ transformed. Individual error bars for each strain are not displayed for clarity purposes. Colours represent different serotypes (as in Figure 1). Different shapes are used to distinguish

960 strains from the same serotype. B. Minimum generation time of the mutant and the wild type strains relative to the parental strain in each environment. C. Maximum yield of the mutant and the wild type strains relative to the parental strain in each environment. Statistics were calculated using a paired t-test. $* \mathrm{P}<0.05$.

965 Figure 4. Fate of capsule mutants in host-related context. A. Resistance to human serum. Data is presented as relative survival to control (heat-inactivated human serum). $* \mathrm{P}<0.05 ; * *$ $\mathrm{P}<0.01 ; * * * \mathrm{P}<0.001$ paired t-test. Only significant statistics are represented. B. Correlation between the total amount of biofilm formed by the wild type and the difference in biofilm formation between the wild type and the deletion mutant across environments. P-values 
into account the five strains with no off-target mutations. Absolute values of biofilm formation are shown in Figure S9.

Figure 5. The environment, not the serotype, explains most of the variance observed across capsule-associated traits. A. Principal component analyses (PCA) of capsule-related phenotypes. Phenotypes are coloured by environment. Each individual point represents a strain. PCA was performed using the prcomp function (options scale and center $=$ TRUE) in $\mathrm{R}$ and we used the ggbiplot package for visualization. B. Correlation matrix of all traits. Numbers indicate Spearman's correlation. Stars identify significant correlations $(\mathrm{P}<0.05)$. 


\section{SUPPLEMENTAL FIGURE LEGENDS}

Figure S1. Capsule quantification of each strain in each growth media by the uronic acid method.

985

Figure S2. Ratio of capsulated colonies throughout 30 days of evolution in two media. Strains were diluted 1:100 and inoculated in fresh media every 24 hours during 30 days. CFUs were counted every day. Each line represents an independent population $(\mathrm{N}=3)$.

990 Figure S3. Capsule quantification of each mutant by the uronic acid method. This experiment was performed on overnight cultures grown in LB. Each dot corresponds to an independent experiment. The background noise of this assay is established around $1.5 \mathrm{ng} / \mu \mathrm{L}$. Statistical tests: $* \mathrm{P}<0.05 ; * * \mathrm{P}<0.01 ; * * * \mathrm{P}<0.001$.

995 Figure S4. Fitness advantage/disadvantage of capsulated strains in nutrient poor/rich media against wza mutants (A) or wcaJ/wbaP mutants (B). Competition between wild type and their isogenic capsule mutants in different growth media. Each point represents the average of at least three independent replicates. ${ }^{*} \mathrm{P}<0.05$ Tukey post hoc, $\mathrm{p}$-values adjusted for multiple testing.

1000

Figure S5. Survival of strains under starvation and desiccation. A. Survival of parental, mutant and wild type strains, relative to day 0. Data is $\log _{10}$-transformed. Error bars are not displayed for clarity purposes. Colours represent different serotypes (as in Figure 1), whereas the shape represent different species. B. Cellular survival, in CFU, after 1, 2 or four days of 1005 desiccation at $37^{\circ} \mathrm{C}$. Error bars for independent points are not shown for clarity purposes.

Figure S6. Growth parameters of the strains used in this study. A. Minimum generation time (A), maximum yield (B) and area under the growth curve $(\mathbf{C})$ of the mutant and wild type strains, relative to the parental strain. Statistics were calculated using a paired t-test. * $\mathrm{P}$ $<0.05$, ** $\mathrm{P}<0.01, * * * \mathrm{P}<0.001$. D. Percentage of dead cells after 16 hours growth in a microtiter plate well. Each symbol represents an independent experiment. No significant differences are found across genotypes. 
1015 Figure S7. $\log _{10}$-increase of CFU after 48 hours of growth. Growth of $\Delta w c a J$ (\#26 and \#56) and $\triangle w b a P(\# 24)$ mutants was followed for 24 and 48 hours. Cells were grown in M63B1 without nutrient source (Control), with $0.8 \mathrm{ng} / \mu \mathrm{L}$ of purified capsule $(\mathrm{Cap}+)$, an equivalent volume of extraction of a non-capsulated strain (Cap-), and $0.8 \mathrm{ng} / \mu \mathrm{L}$ of glucuronic acid (the standard used to quantify the capsule). Individual points represent four

1020 independent experiments. Relevant statistical comparisons are shown, (ANOVA with posthoc Tukey correction for multiple comparisons)

Figure S8. Resistance to bile salts at physiological concentrations $(0.05 \%)$, and at $10 \mathrm{X}$ (0.5\%). Results are presented as $\log _{10}$ (relative to control, LB plates). $* \mathrm{P}<0.05$, $* * \mathrm{P}<0.01$, $* * * \mathrm{P}<0.001$

Figure S9. Biofilm formation of parental, mutant and wild type strains across growth media. $\mathrm{P}$ values reflect paired t-tests. $* \mathrm{P}<0.05, * * \mathrm{P}<0.01, * * * \mathrm{P}<0.001$

1030 Figure S10. Growth rate and maximum yield of each population in each environment.

Figure S11. Biofilm formation of all strains in all environments.

Figure S12.Mucoviscosity index of strains in all environments.

1035

Figure S13. PCA analyses of capsule-related phenotypes. Phenotypes are coloured by serotype. Each individual point represents a strain. PCA was performed using prcomp function in $\mathrm{R}$ and ggbiplot package for visualization purposes. 
bioRxiv preprint doi: https://doi.org/10.1101/2020.04.27.059774; this version posted September 14, 2020. The copyright holder for this preprint (which was not certified by peer review) is the author/funder, who has granted bioRxiv a license to display the preprint in perpetuity. It is made available under aCC-BY-NC-ND 4.0 International license.

\section{FIGURES}

1045

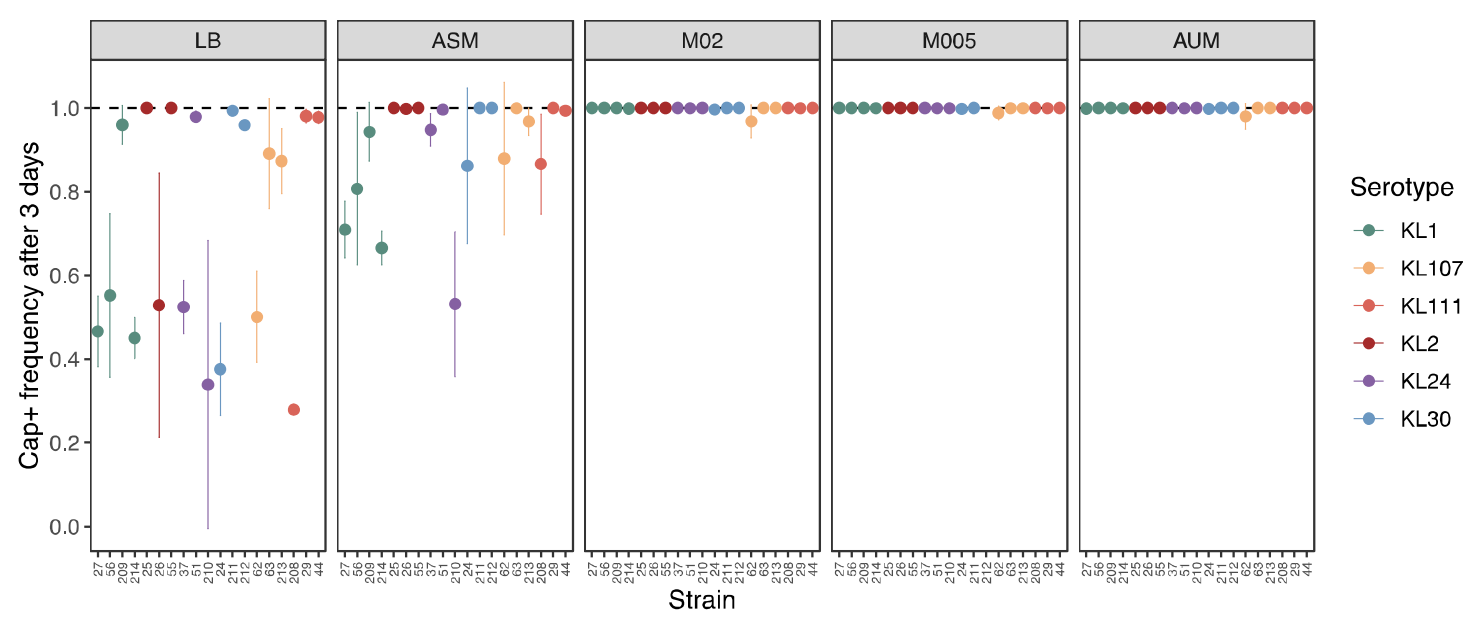

Figure 1 
bioRxiv preprint doi: https://doi.org/10.1101/2020.04.27.059774; this version posted September 14,2020 . The copyright holder for this preprint (which was not certified by peer review) is the author/funder, who has granted bioRxiv a license to display the preprint in perpetuity. It is made available under aCC-BY-NC-ND 4.0 International license.

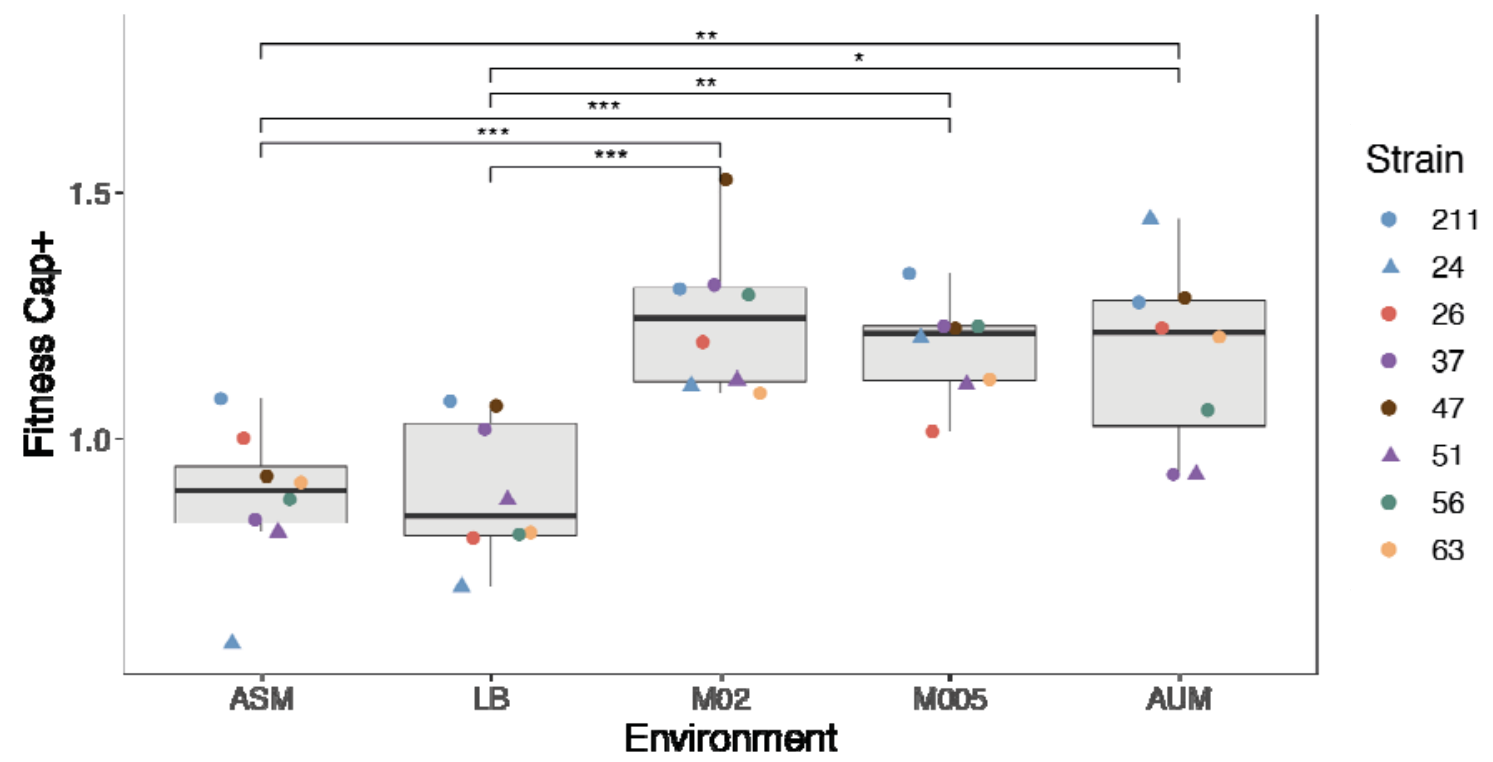

Figure 2 


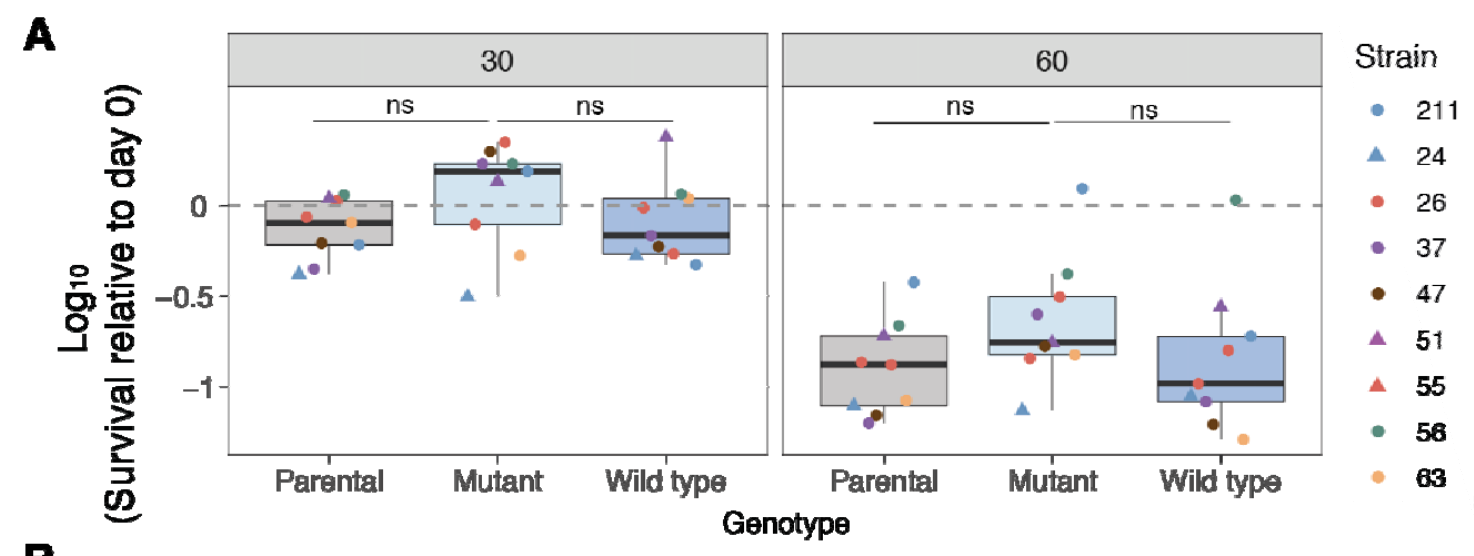

B

$\begin{array}{r}211 \\ \text { - } 24 \\ -26 \\ \text { - } 37 \\ \text { - } 47 \\ \hline \quad 51 \\ \hline 55 \\ \hline 56 \\ \hline\end{array}$


Strain

- 211

$\therefore \quad 24$

- 26

- 37

- 47

$\triangle 51$

- 56

- 63

C

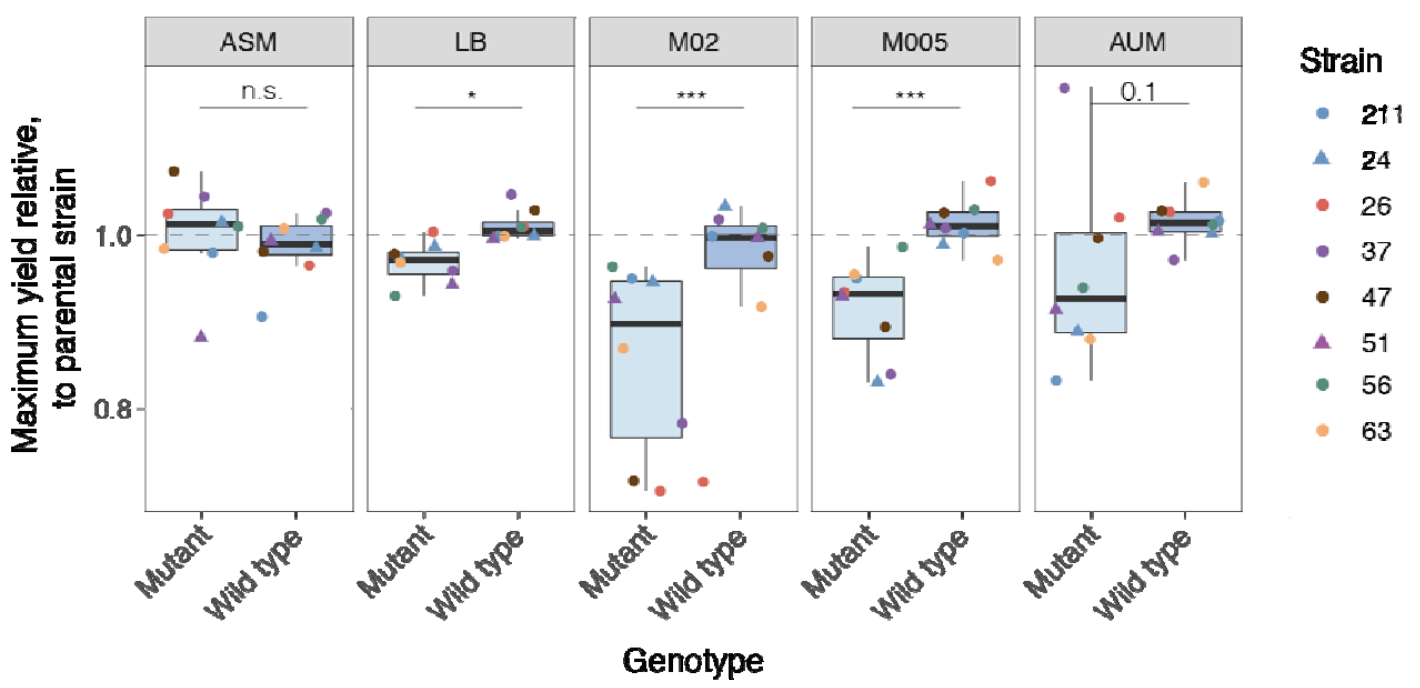

Figure 3 
bioRxiv preprint doi: https://doi.org/10.1101/2020.04.27.059774; this version posted September 14,2020 . The copyright holder for this preprint (which was not certified by peer review) is the author/funder, who has granted bioRxiv a license to display the preprint in perpetuity. It is made available under aCC-BY-NC-ND 4.0 International license.

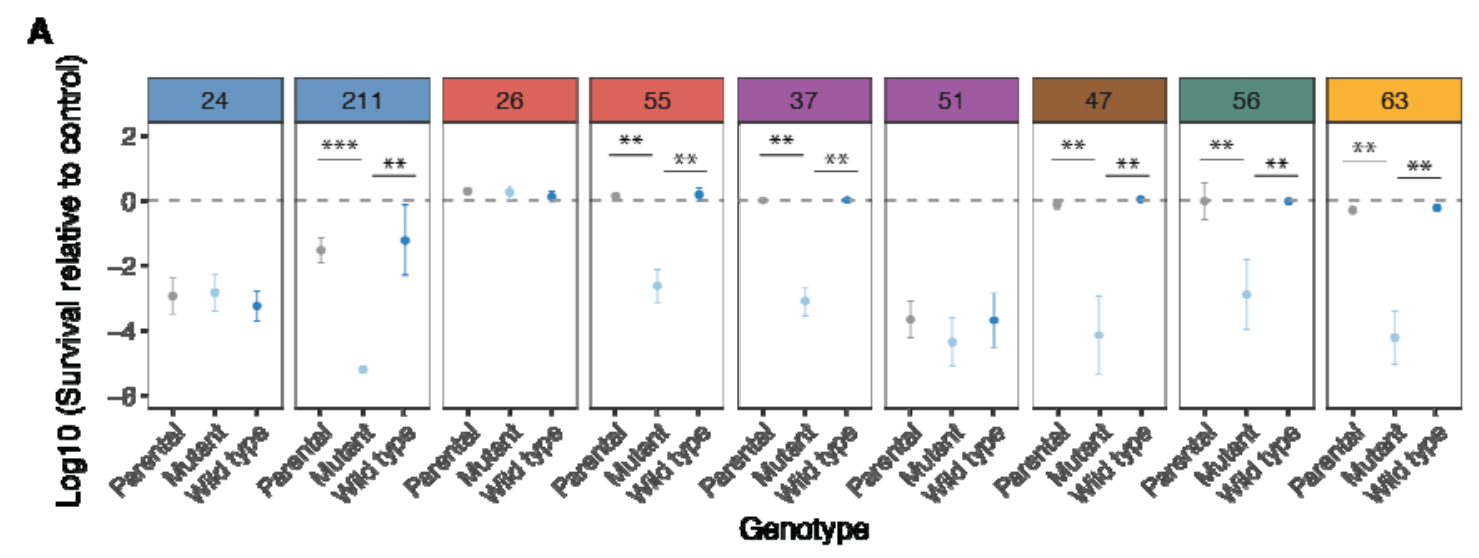

B

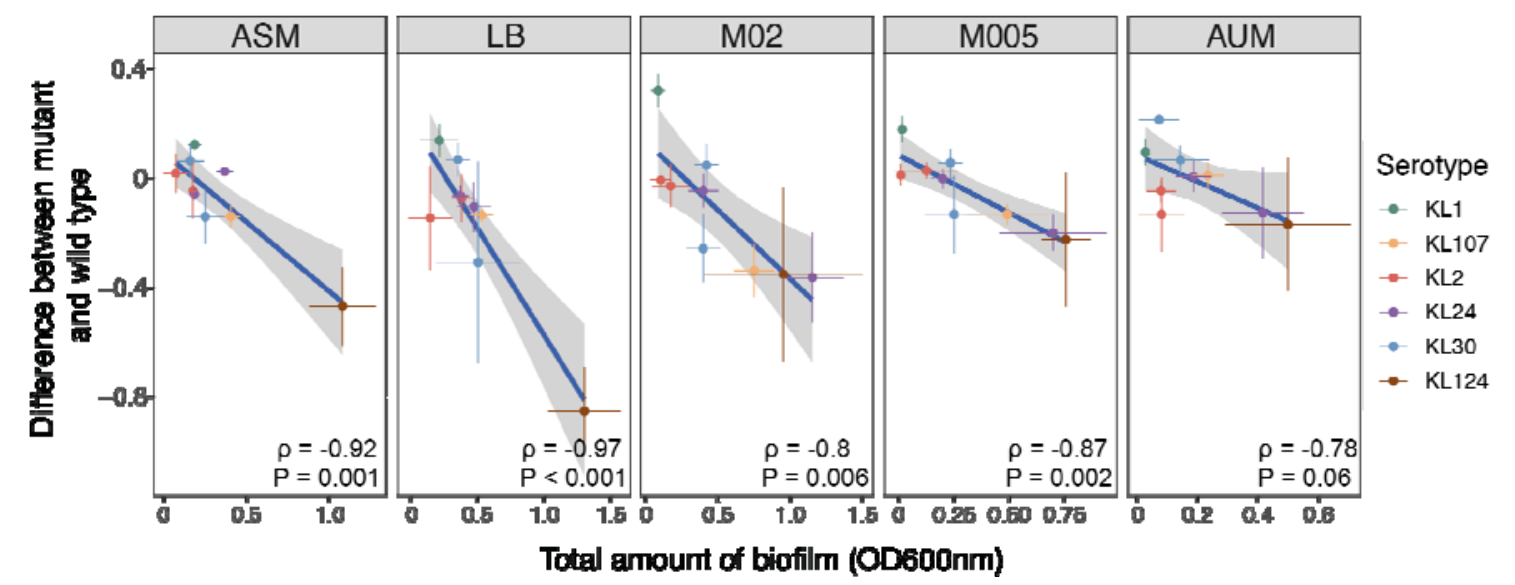

1055

Figure 4 
bioRxiv preprint doi: https://doi.org/10.1101/2020.04.27.059774; this version posted September 14,2020 . The copyright holder for this preprint (which was not certified by peer review) is the author/funder, who has granted bioRxiv a license to display the preprint in perpetuity. It is made available under aCC-BY-NC-ND 4.0 International license.

A

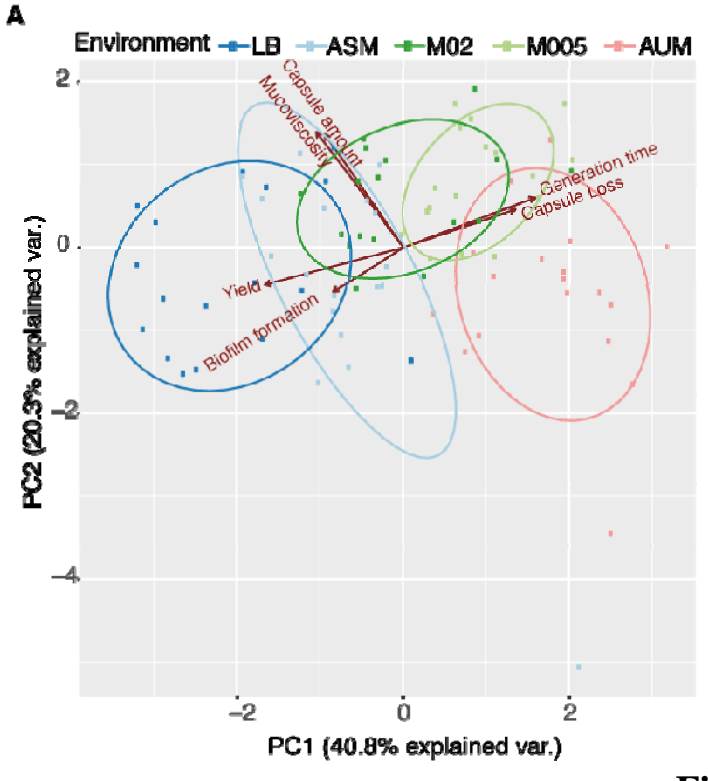

B

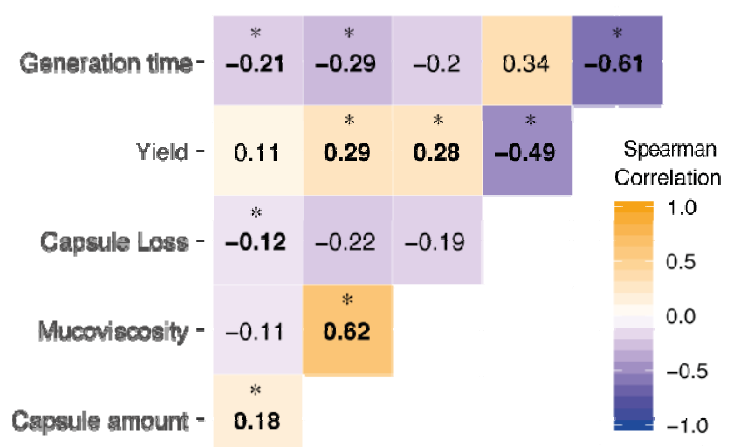

Figure 5 